\title{
EFEITOS DE LATIFOLICIDAS APLICADOS EM PÓS-EMERGÊNCIA SOBRE ALGUMAS CARACTERÍSTICAS AGRONÔMICAS DA SOJA CV. EMGOPA 316
}

\author{
Antônio Carlos de Barros ${ }^{1}$, Pedro M. F. de O. Monteiro ${ }^{1}$, Luiz Cláudio de Faria ${ }^{2}$, \\ José Nunes Júnior ${ }^{3}$, Xavier C. Furtado ${ }^{3}$ e Renato A. Pinto ${ }^{4}$
}

\author{
Engenheiro Agrônomo, Pesquisador. Agencia Rural-GO. Caixa Postal 314. Jataí, GO 75800-069 \\ ${ }^{2}$ Engenheiro Agrônomo, Pesquisador. Embrapa Soja/CTPA. Caixa Postal 533. Goiânia, GO 74001-970 \\ ${ }^{3}$ Engenheiro Agrônomo, Pesquisador. CTPA. Caixa Postal 533. Goiânia, GO 74001-970 \\ ${ }^{4}$ Engenheiro Agrônomo, Pesquisa e Desenvolvimento de Produtos. AVENTIS. Caixa Postal 7. Paulínia, SP 13140-000
}

\begin{abstract}
RESUMO
Conduziu-se um ensaio na fazenda Bela Vista, município de Serranópolis-GO, objetivando avaliar os efeitos de latifolicidas aplicados em pós-emergência, sobre algumas características agronômicas da soja cv. Emgopa 316, com ciclo de maturação precoce. Os tratamentos testados foram: lactofen (168 g/ha), chlorimuron-ethyl (15 g/ha) + óleo mineral (0,2 $\mathrm{v} / \mathrm{v})$, fomesafen $(250 \mathrm{~g} / \mathrm{ha})+$ óleo mineral $(0,2 \mathrm{v} / \mathrm{v})$, imazethapyr $(100 \mathrm{~g} / \mathrm{ha})+$ óleo mineral $(0,2 \% \mathrm{v} / \mathrm{v})$, chlorimuronethyl+lactofen $(12,5+96 \mathrm{~g} / \mathrm{ha})$, chlorimuron-ethyl+fomesafen $(12,5+150 \mathrm{~g} / \mathrm{ha})$, chlorimuron-ethyl+imazethapyr $(12,5+50$ $\mathrm{g} / \mathrm{ha})$, imazethapyr+lactofen $(50+96 \mathrm{~g} / \mathrm{ha})$, imazethapyr+fomesafen $(50+150 \mathrm{~g} / \mathrm{ha})$, além da testemunha. Os herbicidas, nas doses estudadas, provocaram injúrias às plantas de soja cv. Emgopa 316, com maior intensidade nos tratamentos contendo lactofen, porém não influenciaram na altura das plantas aos 15, 30 e 45 DAT e na colheita da soja. Também não afetaram a altura de inserção da primeira vagem, densidade de plantas, número de vagens/planta, peso de 1000 sementes e nem o rendimento de grãos. A mistura imazethapyr+fomesafen $(50+150 \mathrm{~g} / \mathrm{ha})$ proporcionou o maior número de entrenós, porém, não diferiu estatisticamente dos demais tratamentos, exceto para lactofen ( $168 \mathrm{~g} / \mathrm{ha})$, que proporcionou o menor número de entrenós. O herbicida lactofen isolado ou em combinação com chlorimuron-ethyl ou com imazethapyr, pode se utilizado em soja cv. Emgopa 316, com ciclo de maturação precoce, apresentando resultados semelhantes ao fomesafen isolado ou em mistura com chlorimuron-ethyl ou imazethapyr.
\end{abstract}

Palavras-chave: actofen, fomesafen, imazethapyr, chlorimuron-ethyl, fitotoxicidade.

\section{ABSTRACT \\ Effect of postemergence herbicides on agronomics characteristics of soybean cv. Emgopa 316}

A experiment was carried out at the Bela Vista Farm, in Serranópolis, State of Goiás, with the objective to evaluate the effects of postemergence herbicides on agronomic characteristcs of soybean cv. Emgopa 316, with premature maturation cycle. Treatments consisted of lactofen $(168 \mathrm{~g} / \mathrm{ha})$, chlorimuron-ethyl $(15 \mathrm{~g} / \mathrm{ha})+$ mineral oil $(0.2 \mathrm{v} / \mathrm{v})$, fomesafen $(250 \mathrm{~g} / \mathrm{ha})+$ mineral oil $(0.2 \mathrm{v} / \mathrm{v})$, imazethapyr (100 g/ha), chlorimuron-ethyl+lactofen (12.5+96 g/ha), chlorimuronethyl+fomesafen $(12.5+150 \mathrm{~g} / \mathrm{ha})$, chlorimuron-ethyl+imazethapyr $(12.5+50 \mathrm{~g} / \mathrm{ha})$, imazethapyr+lactofen $(50+96 \mathrm{~g} / \mathrm{ha})$, imazethapyr+fomesafen $(50+150 \mathrm{~g} / \mathrm{ha})$, and a handweeded control. The studied herbicide doses caused some injury to soybean, with more intensity in the treatments with lactofen. However, they did not significantly influence the height of plants at 15, 30 and 45 days after treatments and at soybean harvest. The herbicides also did not significantly affect 
insertion height of the first pod, final stand, number of pods/plant, weight of 1000 seeds and grain production. Imazethapyr+fomesafen $(50+150 \mathrm{~g} / \mathrm{ha})$ gave the best result in number of internodes, but it did not differ statistically from the other treatments, except for lactofen $(168 \mathrm{~g} / \mathrm{ha})$, that proportioned the lowest number of internodes. Lactofen isolated or combined with chlorimuron-ethyl or with imazethapyr can be used in soybean cv. Emgopa 316, showing results similar to fomesafen isolated or in mixture with chlorimuron-ethyl or imazethapyr.

Key words: lactofen, fomesafen, imazethapyr, chlorimuron-ethyl, phytotoxicity.

\section{INTRODUÇ̃̃O}

Chlorimuron-ethyl e imazethapyr são herbicidas seletivos para cultura da soja, recomendados para o controle de plantas daninhas dicotiledôneas. Embora sejam de grupos químicos diferentes, apresentam modos de ação semelhantes, que consiste na inibição da enzima acetolactato sintase (ALS) (Vidal, 1997). Esta enzima cataliza reações de síntese dos aminoácidos valina, leucina e isoleucina. A redução dos níveis de aminoácidos acarreta sérios distúrbios na produção de proteínas, interferindo no crescimento celular, provocando necrose no meristema apical e paralização do crescimento dos mesmos (Fleck \& Vidal, 1994).

Fomesafen e lactofen são herbicidas do grupo químico difenil éter, utilizados em pós-emergência, no controle seletivo de plantas daninhas dicotiledôneas na cultura da soja. Estes produtos são inibidores da enzima protox (Vidal, 1997), causando nas plantas de soja clorose e necrose dos tecidos atingidos. O lactofen pode ocasionar, também, enrugamento dos folíolos que se encontram em desenvolvimento na época da aplicação e redução na altura das plantas (Sheperd et al., 1984, citado por Damião et al., 1992).

Há uma preocupação dos agricultores e técnicos, com os possíveis efeitos tóxicos dos herbicidas sobre o desenvolvimento e produção das culturas. No entanto, Velini et al. (1992), relatam que a seletividade e a capacidade de um determinado herbicida eliminar

plantas daninhas infestantes de uma cultura, sem reduzir a produtividade e a qualidade do produto final colhido, não pode ser determinada apenas pela verificação ou não de sintomas de intoxicação. Já são conhecidos exemplos de herbicidas que podem reduzir a produtividade das culturas sem manifestar efeitos visualmente destacáveis, e também de herbicidas que causam injúrias bastante acentuadas, mas que permitem às culturas manifestar suas reais potencialidades produtivas.

O objetivo do presente trabalho foi avaliar os efeitos de intoxicação de latifolicidas aplicados em pós-emergência, sobre algumas características agronômicas da soja cv. Emgopa 316, com ciclo de maturação precoce.

\section{MATERIAIS E MÉTODOS}

O experimento foi conduzido na fazenda Bela Vista, localizada no município de Serranópolis-GO, região fisiográfica Serra do Caiapó (Sudoeste de Goiás).

No dia 29/09/99, foi efetuada a dessecação da vegetação daninha, utilizando-se o herbicida glyphosate (1080 g/ha) e no dia 01/10/99 foi realizada a semeadura da soja cv. Emgopa 316 , recebendo nos sulcos de plantio a adubação de $350 \mathrm{~kg} /$ ha, da fórmula 02-20-18.

O delineamento experimental adotado foi de blocos ao acaso, com cinco repetições e dez tratamentos, relacionados na Tabela 1. Os herbicidas foram aplicados em pós-emergência em 24/10/99, quando a soja apresentava-se com três trifólios. No momento da aplicação a temperatura do ar era de $26^{\circ} \mathrm{C}$, e a umidade relativa de $72 \%$, sendo que a pulverização ocorreu das 08:45 às 09:15 h. Para aplicação dos produtos foi utilizado um pulverizador costal, pressurizado a $\mathrm{CO}_{2}$, pontas de pulverização (bicos) tipo leque, série TT 110.02, calibrados para $3,0 \mathrm{~kg} / \mathrm{cm}^{2}$ de pressão, consumindo $200 \mathrm{l} / \mathrm{ha}$ de calda.

Para evitar possíveis interferências das plantas daninhas, as parcelas experimentais, foram mantidas no limpo durante todo o ciclo da cultura, realizando-se três capinas manuais. As parcelas experimentais mediram 7,0 $\mathrm{m} \times 3,0 \mathrm{~m}\left(21 \mathrm{~m}^{2}\right)$, com a área útil, para observações, de 5,0 $\mathrm{m}$ x 1,0 m $\left(5,0 \mathrm{~m}^{2}\right)$.

As precipitações pluviais registradas no período de outubro/99 a janeiro/2000, encontram-se discriminadas na Tabela 2 .

A fitotoxicidade provocada pelos herbicidas foi avaliada aos 8, 15 e 30 dias após a aplicação dos tratamentos (DAT), adotando-se a escala visual em percentagem, sendo que 0 (zero) corresponde à ausência de intoxicação e 100 (cem) a dano total à cultura.

Aos 15, 30 e 45 DAT foram efetuadas as medidas de altura de plantas de soja e no final do ciclo da cultura, as medidas da altura de inserção da primeira vagem, contagem do número de vagens/planta, número de entrenós e densidade de plantas de soja. Foram avaliados também o peso de 1000 sementes e o rendimento de grãos.

Exceto para toxicidade, os resultados foram submetidos à análise de variância e as médias comparadas pelo teste de Tukey, ao nível de $5 \%$ de probabilidade. 
Tabela 1. Herbicidas utilizados com as respectivas doses do produto comercial e do ingrediente ativo. Serranópolis, GO, 1999/2000.

\begin{tabular}{|c|c|c|c|}
\hline \multicolumn{2}{|r|}{ Tratamento } & \multicolumn{2}{|c|}{ Dose } \\
\hline $\begin{array}{l}\text { Produto comercial } \\
\text { (p.c) }\end{array}$ & $\begin{array}{l}\text { Ingrediente ativo } \\
\text { (i.a) }\end{array}$ & $\begin{array}{c}\text { p.c } \\
(\mathrm{g} 0 \mathrm{u} / / \mathrm{ha})\end{array}$ & $\begin{array}{c}\text { i.a } \\
(\mathrm{g} / \mathrm{h} \mathrm{a})\end{array}$ \\
\hline Classic $^{1}$ & Chlorimuron-ethyl & $60 \mathrm{~g}$ & 15 \\
\hline Cobra & Lactofen & 0,71 & 168 \\
\hline Flex $^{1}$ & Fomes ofen & 1,01 & 250 \\
\hline Pivot & Imazethapyr & 1,01 & 100 \\
\hline Classic + cobra & Chlorimuron-ethy $1+$ lactofen & $50 \mathrm{~g}+0,4 \mid$ & $125+96$ \\
\hline Classic + flex ${ }^{1}$ & Chlorimuron-ethy $1+$ fomesafen & $50 \mathrm{~g}+0,61$ & $125+150$ \\
\hline Classic + pivot & Chlorimuron-ethyl+imazethapyr & $50 \mathrm{~g}+0,5$ & $125+50$ \\
\hline Pivot+cobra & Imazethapyr+lactofen & $0,5|+0,4|$ & $50+96$ \\
\hline Pivot + flex & Imazethapyr+fomesafen & $0,5|+0,6|$ & $50+150$ \\
\hline Testemunha & - & - & - \\
\hline
\end{tabular}

'Foi adicionado à calda de pulverização o adjuvante ASSIST $(0,2 \%$ v/v).

Tabela 2. Precipitações pluviais diá rias $(\mathrm{mm})$ registradas no período de outubro de 1999 a janeiro de 2000. Serranópolis, GO, 1999/2000.

\begin{tabular}{|c|c|c|c|c|}
\hline Dias & Outubro & Novembro & Dezembro & Janeir o \\
\hline 1 & 0.0 & 0.0 & 0.0 & 0.0 \\
\hline 2 & 0.0 & 0.0 & 0.0 & 28.8 \\
\hline 3 & 0.0 & 0.3 & 18.6 & 83 \\
\hline 4 & 0.0 & 45.0 & 0.0 & 65.6 \\
\hline 5 & 0.0 & 7.4 & 0.1 & 0.0 \\
\hline 6 & 3.1 & 0.0 & 0.3 & 5.4 \\
\hline 7 & 0.0 & 0.0 & 0.0 & 3.1 \\
\hline 8 & 1.4 & 0.0 & 14.9 & 1.5 \\
\hline 9 & 0.0 & 0.4 & 12.5 & 0.0 \\
\hline 10 & 0.0 & 1.2 & 64.3 & 0.0 \\
\hline 11 & 0.0 & 0.0 & 1.4 & 0.0 \\
\hline 12 & 0.0 & 0.0 & 23.1 & 0.0 \\
\hline 13 & 0.0 & 32.5 & 0.5 & 4.8 \\
\hline 14 & 11.8 & 15.3 & 0.0 & 0.0 \\
\hline 15 & 0.0 & 0.0 & 3.4 & 0.0 \\
\hline 16 & 0.0 & 0.0 & 0.0 & 0.0 \\
\hline 17 & 0.0 & 0.0 & 0.0 & 0.0 \\
\hline 18 & 1.6 & 0.0 & 0.0 & 0.4 \\
\hline 19 & 3.0 & 0.0 & 0.0 & 0.0 \\
\hline 20 & 0.0 & 0.0 & 0.0 & 4.2 \\
\hline 21 & 0.0 & 41.9 & 0.0 & 26 \\
\hline 22 & 0.0 & 0.0 & 1.1 & 0.2 \\
\hline 23 & 0.0 & 6.4 & 0.0 & 1.6 \\
\hline 24 & 0.0 & 3.2 & 1.1 & 0.0 \\
\hline 25 & 4.1 & 3.2 & 0.0 & 4.2 \\
\hline 26 & 0.0 & 8.8 & 13.0 & 22 \\
\hline 27 & 47.2 & 16.7 & 0.0 & 21 \\
\hline 28 & 1.3 & 1.2 & 35.4 & 1.6 \\
\hline 29 & 0.0 & 0.0 & 0.0 & 1.7 \\
\hline 30 & 0.4 & 0.0 & 8.4 & 0.0 \\
\hline 31 & 0.0 & - & 31.6 & 0.9 \\
\hline TOTAL & 101.8 & 183.5 & 229.7 & 283.5 \\
\hline
\end{tabular}

\section{RESULTADOS E DISCUSSÃO}

Os herbicidas lactofen (168 g/ha), chlorimuronethyl+lactofen $(12,5+96 \mathrm{~g} / \mathrm{ha})$ e imazethapyr+lactofen $(50+96$ $\mathrm{g} / \mathrm{ha}$ ), proporcionaram os maiores níveis de intoxicação às plantas de soja, aos 8 DAT, porém dentro de limites aceitáveis na prática. Fomesafen $(250 \mathrm{~g} / \mathrm{ha})+$ óleo mineral $(0,2 \% \mathrm{v} / \mathrm{v})$, chlorimuron-ethyl+fomesafen $(12,5+150 \mathrm{~g} / \mathrm{ha})+$ óleo mineral $(0,2 \% \mathrm{v} / \mathrm{v})$; imazethapyr+fomesafen $(50+150 \mathrm{~g} / \mathrm{ha})$, chlorimuron-ethyl $(15,0 \mathrm{~g} / \mathrm{ha})+$ óleo mineral $(0,2 \% \mathrm{v} / \mathrm{v})$; chlorimuron-ethyl+imazethapyr $(12,5+50 \mathrm{~g} / \mathrm{ha})$ e imazethapyr (100 g/ha), também provocaram injúrias iniciais visíveis às plantas de soja, porém com índices inferiores. Tais sintomas foram diminuindo com o passar do tempo, e aos 30 DAT a cultura da soja apresentava-se totalmente recuperada em todas as parcelas tratadas com estes produtos (Tabela 3 ).

Nos tratamentos contendo lactofen ou fomesafen foram observados sintomas de clorose e necrose nas folhas de soja, porém a folhagem nova emitida após a aplicação dos produtos, não apresentava sintomas visíveis de intoxicação, concordando com Vidal (1997). Podem ocasionar, também, enrrugamento dos folíolos que se encontravam em desenvolvimento na época da aplicação e redução na altura de plantas (Sheperd et al., 1984, citado por Damião Filho et al., 1992).

Os sintomas de toxicidade observados na cultura da soja, causados pelos herbicidas chlorimuron-ethyl, imazethapyr ou a mistura em tanque dos dois produtos, foram manifestados pela descoloração das folhas novas e pequenas inibição do crescimento inicial da soja, concordando com Vidal (1997) e Leite et al. (1998).

Melhorança \& Pereira (1999) também observaram sintomas visíveis de fitotoxicidade à cultura da soja, com a aplicação de lactofen, lactofen+imazethapyr, lactofen+ chlorimuron-ethyl e imazethapyr. 
Tabela 3. Resultados médios de toxicidade, altura de planta aos 15,30 e 45 DAT e na colheita, número de entrenós e número de vagens/planta, obtidos no ensaio com latifolicidas de pós-emergência. Serranópolis,GO, 1999/2000.

\begin{tabular}{|c|c|c|c|c|c|c|c|c|c|c|}
\hline \multirow[b]{2}{*}{ Tratamento } & \multirow[b]{2}{*}{$\begin{array}{l}\text { Dose } \\
\text { (g/ha) }\end{array}$} & \multicolumn{3}{|c|}{ Toxicidade } & \multicolumn{4}{|c|}{ Altura de plantas ${ }^{2}(\mathrm{~cm})$} & \multirow{2}{*}{$\begin{array}{l}\text { Número de } \\
\text { entrenós }\end{array}$} & \multirow{2}{*}{$\begin{array}{c}\text { Número de } \\
\text { vagens } \\
\text { planta }\end{array}$} \\
\hline & & 8DAT & $15 \mathrm{DAT}$ & $30 \mathrm{DAT}$ & $15 \mathrm{DAT}$ & 30 DAT & $45 \mathrm{DAT}$ & $\begin{array}{c}\mathrm{Na} \\
\text { colheita }\end{array}$ & & \\
\hline Chlorimuron-ethyl ${ }^{1}$ & 15 & 8 & 6 & 0 & $24,5 \mathrm{a}$ & $46,8 \mathrm{a}$ & $112,5 \mathrm{a}$ & $116,2 \mathrm{a}$ & $17,2 \mathrm{ab}$ & $34,0 a^{3}$ \\
\hline Lactofen & 168 & 18 & 12 & 0 & $24,8 \mathrm{a}$ & $50,2 \mathrm{a}$ & $113,5 \mathrm{a}$ & $115,7 \mathrm{a}$ & $16,9 b$ & $35,3 \mathrm{a}$ \\
\hline Fomesafen ${ }^{1}$ & 250 & 12 & 5 & 0 & $26,0 \mathrm{a}$ & $51,2 \mathrm{a}$ & $116,5 \mathrm{a}$ & $118,5 \mathrm{a}$ & $18,4 \mathrm{ab}$ & $35,1 \mathrm{a}$ \\
\hline Imazethapyr & 100 & 8 & 8 & 0 & $25,0 \mathrm{a}$ & $50,2 a$ & $113,5 \mathrm{a}$ & $116,0 \mathrm{a}$ & $18,6 \mathrm{ab}$ & $37,8 \mathrm{a}$ \\
\hline Chlorimuron-ethyl+lactofen & $12,5+96$ & 18 & 12 & 0 & $25,5 \mathrm{a}$ & $52,0 \mathrm{a}$ & $113,8 \mathrm{a}$ & $115,7 \mathrm{a}$ & $18,3 \mathrm{ab}$ & $40,1 \mathrm{a}$ \\
\hline Chlorimuron-ethyl+fomesafen 1 & $12,5+150$ & 14 & 8 & 0 & $26,2 \mathrm{a}$ & $50,0 \mathrm{a}$ & $115,2 \mathrm{a}$ & $116,5 \mathrm{a}$ & $17,7 \mathrm{ab}$ & $39,4 \mathrm{a}$ \\
\hline Chlorimuron-ethyl+imazethapyr ${ }^{3}$ & $12,5+50$ & 15 & 10 & 0 & $25,2 a$ & $51,2 \mathrm{a}$ & $112,0 \mathrm{a}$ & $116,7 \mathrm{a}$ & $17,6 \mathrm{ab}$ & 33,3 a \\
\hline Imazethapyr+ladofen & $50+96$ & 18 & 12 & 0 & $24,8 \mathrm{a}$ & $49,8 \mathrm{a}$ & $113,0 \mathrm{a}$ & $117,0 \mathrm{a}$ & $17,0 \mathrm{ab}$ & $35,0 \mathrm{a}$ \\
\hline Imazethapyr+formesafen & $50+150$ & 14 & 10 & 0 & $25,0 \mathrm{a}$ & $50,8 \mathrm{a}$ & $112,8 \mathrm{a}$ & $117,7 \mathrm{a}$ & $19,2 \mathrm{a}$ & $38,1 \mathrm{a}$ \\
\hline Testemunha & & 0 & 0 & 0 & $24,5 \mathrm{a}$ & $50,7 \mathrm{a}$ & $115,2 \mathrm{a}$ & $120,7 \mathrm{a}$ & $18,1 \mathrm{ab}$ & $39,5 \mathrm{a}$ \\
\hline C.V. (\%) & & & & & 5,2 & 5,5 & 2,6 & 2,3 & 5,0 & 14,1 \\
\hline $\mathrm{DMS}$ & & & & & 3,15 & 6,74 & 7,21 & 5,75 & 2,17 & 14,10 \\
\hline
\end{tabular}

${ }^{\mathrm{t}}$ Foi adicionado à calda de pulverização óleo mineral a $0,2 \% \mathrm{v} / \mathrm{v}$

${ }^{2}$ Média de 10 plantas

${ }^{3}$ Médias na coluna seguidas das mesmas letras, não diferem significativamente entre si, pelo teste de Tukey a 5\% de probabilidade.

Barros (1995), avaliando a resposta de quatro cultivares de soja, com ciclos de maturação precoce a semi-precoce, frente aos herbicidas lactofen, chlorimuron-ethyl+lactofen, fomesafen, chlorimuron-ethyl+fomesafen, dentre outros, observou sintomas iniciais de intoxicação em todas as cultivares de soja, com recuperação total aos 35 DAT, concordando com os resultados deste trabalho de pesquisa.

Não foram observadas diferenças estatisticamente significativas entre as alturas de plantas avaliadas aos 15,30 e 45 DAT, nos diferentes tratamentos químicos e testemunha (Tabela 3 ).

A aplicação dos herbicidas também, não afetou significativamente a altura de plantas no final do ciclo da soja e nem o número de entrenós e número de vagens/planta da cultivar Emgopa 316.

Avaliando a resposta dos cultivares Garimpo, FT Estrela, FT Eureka e Emgopa 302, frente aos herbicidas lactofen, fomesafen, bentazon e misturas deles com o chlorimuron-ethyl, também não foram observadas diferenças significativas entre os tratamentos químicos e a testemunha quanto a altura de plantas e inserção da primeira vagem (Barros, 1995).

A densidade de plantas final, a altura de inserção da primeira vagem, o peso de 1000 sementes e o rendimento de grãos de soja não foram influenciados significativamente pela aplicação dos tratamentos (Tabela 4).

Apesar de não terem sido observadas diferenças significativas entre as produtividades da soja, o menor rendimento de grãos foi obtido na testemunha, possivelmente devido à danificação do sistema radicular da soja, o que ocasionou pequena redução na produtividade da cultura. No entanto, o número de entrenós no tratamento contendo imazethapyr+ fomesafen $(50+150 \mathrm{~g} / \mathrm{ha})$ foi semelhante estatisticamente aos outros tratamentos químicos e à testemunha, exceto para lactofen (168 g/ha), que proporcionou o menor número de entrenós (Tabela 3 ).

A mistura de imazethapyr com chlorimuron-ethyl proporcionou resultados semelhantes aos outros tratamentos químicos, porém não é aconselhável o uso desta mistura, pois ela contribui para o aparecimento de resistência de plantas daninhas aos herbicidas inibidores da enzima ALS (Vidal, 1997; Leite et al., 1998).

As precipitações pluviais registradas durante o ciclo da cultura foram consideradas normais para a região fisiográfica Serra do Caiapó, onde foi instalado o experimento. Apenas na fase inicial do desenvolvimento da soja houve pequena irregularidade na distribuição das chuvas (Tabela 2), sem, contudo, afetar a emergência e o crescimento desta cultura.

Analisando os resultados no conjunto verifica-se que a aplicação de lactofen isolado ou em combinação com chlorimuron-ethyl ou com imazethapyr, proporcionaram níveis de intoxicação superiores àqueles obtidos pelo fomesafen isolado ou na mistura em tanque com chlorimuron-ethyl e imazethapyr, além de chlorimuron-ethyl+imazethapyr, não afetando, porém, significativamente a altura de plantas aos 15, 30 e 45 DAT e na colheita da soja. Também não tiveram influências significativas na altura de inserção da primeira vagem, densidade de plantas final, número de vagens/planta, peso de 1000 sementes e rendimento de grãos. Somente o número de entrenós no tratamento imazethapyr+fomesafen $(50+150 \mathrm{~g} / \mathrm{ha})$ foi superior estatisticamente àquele obtido no tratamento com lactofen (168 g/ha).

Observa-se, portanto, que os níveis de intoxicação na soja cv. Emgopa 316, provocados pelo herbicida lactofen isolado ou em combinação com chlorimuron-ethyl ou com 
Tabela 4. Resultados médios das alturas de inserção da primeira vagem, densidade de plantas de soja, peso de 1000 sementes e rendimento de grãos, obtidos no ensaio com latifolicidas de pós-emergência. Serranópolis,GO, 1999/2000.

\begin{tabular}{|c|c|c|c|c|c|}
\hline Tratamento & $\begin{array}{l}\text { Dose } \\
\text { (g/ha) }\end{array}$ & $\begin{array}{c}\text { Altura de } \\
\text { inserção da } \\
\text { primeira } \\
\text { vagem }^{2}(\mathrm{~cm})\end{array}$ & $\begin{array}{c}\text { Densidade } \\
\text { de } \\
\text { plantas } / \mathrm{m}^{2}\end{array}$ & $\begin{array}{l}\text { Peso de } 1000 \\
\text { sementes (g) }\end{array}$ & $\begin{array}{l}\text { Rendimento de } \\
\text { grãos (kg/ha) }\end{array}$ \\
\hline 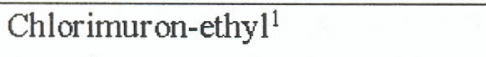 & 15 & $29,5 \mathrm{a}$ & 31 a & $172,2 \mathrm{a}$ & $3823 \mathrm{a}^{3}$ \\
\hline Lactofen & 168 & $28,7 \mathrm{a}$ & 33 a & $170,7 \mathrm{a}$ & 3664 a \\
\hline Fomesafen ${ }^{1}$ & 250 & $34,7 \mathrm{a}$ & 33 a & $168,5 \mathrm{a}$ & 3653 a \\
\hline Imazethapyr & 100 & $29,0 \mathrm{a}$ & $31 \mathrm{a}$ & $164,7 \mathrm{a}$ & $3793 \mathrm{a}$ \\
\hline Chlorimuron-ethyl+lactofen & $12,5+96$ & $27,7 \mathrm{a}$ & $32 \mathrm{a}$ & $168,5 \mathrm{a}$ & $3874 \mathrm{a}$ \\
\hline Chlorimuron-ethyl+fomesafen ${ }^{1}$ & $12,5+150$ & $30,2 \mathrm{a}$ & $33 \mathrm{a}$ & $171,6 \mathrm{a}$ & 3765 a \\
\hline Chlorimuron-ethyl+imazethapyr ${ }^{1}$ & $12,5+50$ & $32,0 \mathrm{a}$ & $33 \mathrm{a}$ & $169,6 \mathrm{a}$ & 3811 a \\
\hline Imazethapyr+lactofen & $50+96$ & $29,0 \mathrm{a}$ & $33 \mathrm{a}$ & $168,9 \mathrm{a}$ & $3741 \mathrm{a}$ \\
\hline Imazethapyr+fomesafen & $50+150$ & 31,5 a & $32 \mathrm{a}$ & $171,2 \mathrm{a}$ & $3654 \mathrm{a}$ \\
\hline Testemunha & & $31,7 \mathrm{a}$ & $31 \mathrm{a}$ & $167,3 \mathrm{a}$ & 3578 a \\
\hline C.V.(\%) & & 7,0 & 13,2 & 3,4 & 9,6 \\
\hline DMS & & 5,1 & 10,2 & 14,1 & 314,2 \\
\hline F (Tratamentos) & & 2,13 n.s & $0,61 \mathrm{n} . \mathrm{s}$ & $0,86 \mathrm{n} . \mathrm{s}$ & $0,93 \mathrm{n} . \mathrm{s}$ \\
\hline
\end{tabular}

${ }^{1}$ Foi adicionado à calda de pulverização óleo mineral Assist $(0,2 \% \mathrm{v} / \mathrm{v})$.

${ }^{2}$ Média de 10 plantas.

${ }^{3}$ Médias na coluna seguidas das mesmas letras, não diferem significativamente entre si, pelo teste de Tukey a $5 \%$ de probabilidade.

imazethapyr, não afetaram o rendimento da soja, nem outras características agronômicas, equivalendo-se aos resultados obtidos nos outros tratamentos químicos e a testemunha. Lorenzi et al. (1994), citado por Melhorança (1999), relataram que níveis significativos de injúrias podem não causar redução de produtividade, concordando com os resultados obtidos no presente experimento.

\section{CONCLUSÕES}

Lactofen aplicado isolado ou em combinação com chlorimuron-ethyl ou com imazethapyr, provocou maiores níveis de intoxicação às plantas de soja, quando comparados ao fomesafen isolado ou na mistura em tanque com chlorimuran-ethyl ou com imazethapyr, ou chlorimuronethyl+imazethapyr, porém, aos 30 DAT a soja apresentava-se totalmente recuperada.

A aplicação dos tratamentos não afetou significativamente a altura das plantas de soja aos 15, 30 e 45 DAT, e na colheita, a inserção da primeira vagem, o número de vagens/ planta, a densidade de plantas final, o peso de 1000 sementes e o rendimento de grãos.

O número de entrenós obtido no tratamento contendo lactofen foi estatisticamente inferior àquele conseguido por imazethapyr+fomesafen, porém foi semelhante aos demais tratamentos químicos e à testemunha.
As injúrias mais elevadas sobre as plantas de soja, provocadas por lactofen isolado ou com combinação com chlorimuron-ethyl ou com imazethapyr, não influenciaram negativamente a produção de grãos e outras características agronômicas da soja, quando comparadas com outros tratamentos químicos e à testemunha, podendo, portanto, serem aplicados à cultivar de soja Emgopa 316, com ciclo de maturação precoce.

\section{LITERATURA CITADA}

BARROS, A. C. de. Resposta de cultivares de soja precoce a latifolicidas pós-emergentes. Goiânia, GO: EMGOPADID, 1995. 14p (EMGOPA. Boletim de Pesquisa, 35).

DAMIÃO FILHO, C. E.; CORSO, G. M.; ANDRADE, V. M. de M. Efeitos do herbicida lactofen sobre três cultivares de soja. I. Alterações morfológicas externas e internas de folhas. Planta Daninha, v. 10, n. 1 -2, p. 17-24, 1992.

FLECK, N. G.; VIDAL, R. A. Injúria potencial de herbicidas de solo ao girassol. III Imazaquin e imazethapyr. Planta Daninha, v. 12, n. 1, p. 39-43, 1994.

LEITE, C. R. F.; ALMEIDA, J. C. V. de.; PRETI, C. E. C. Aspectos fisiológicos, bioquímicos e agronômicos dos herbicidas inibidores da enzima ALS (AHAS). Londrina, PR: Célio R. F. Leite, José C. Vieira de Almeida e Cássio E. C. Preti, 1998. 68 p. 
MELHORANÇA, A. L. Seletividade dos herbicidas diclosulan, flumetsulam e cloransulam em diversas cultivares de soja. In: REUNIÃO DE PESQUISADORES EM CONTROLE DE PLANTAS DANINHAS NOS CERRADOS, 12., 1999, Corumbá-MS. Controle químico de plantas daninhas nos cerrados: ata e anais. Dourados: Embrapa Agropecuária Oeste, 1999.p. 15-18 (Embrapa Agropecuária Oeste. Documentos, 3).

MELHORANÇA, A. L.; PEREIRA, F. A. R. Eficiência do herbicida lactofen no controle de Euphorbia heterophylla resistente aos herbicidas inibidores da enzima acetolactato sintase. In: REUNIÃO DE PESQUISADORES EM CONTROLE DE PLANTAS DANINHAS
NOS CERRADOS, 12., 1999, Corumbá-MS. Controle químico de plantas daninhas nos cerrados: ata e anais. Dourados: Embrapa Agropecuária Oeste, 1999. p. 11-14 (Embrapa Agropecuária. Oeste. Documentos, 3).

VIDAL, R. A. Herbicidas: mecanismos de ação e resistência de plantas. Porto Alegre, RS: Ribas Vidal, 1997.

VELINI, E. D.; FREDERICO, L. A. M.; MORELI, J. L.; MARUBAUYSHI, O. M. Avaliações dos efeitos do herbicida clomazone aplicado em pós-emergência inicial sobre o crescimento e produtividade de soqueira de canade-açúcar (Sacharum officinarum cv. SP. 71. 1406). STAB, Piracicaba, v.10, n.4, p.13-16, 1992. 